\title{
Prior Beliefs Modulate Projection
}

\author{
Judith Degen ${ }^{1}$ and Judith Tonhauser ${ }^{2}$ \\ ${ }^{1}$ Department of Linguistics, Stanford University \\ ${ }^{2}$ Department of English Linguistics, University of Stuttgart
}

Cognitive Science

Keywords: experimental semantics, experimental pragmatics, projection

an open access fournal

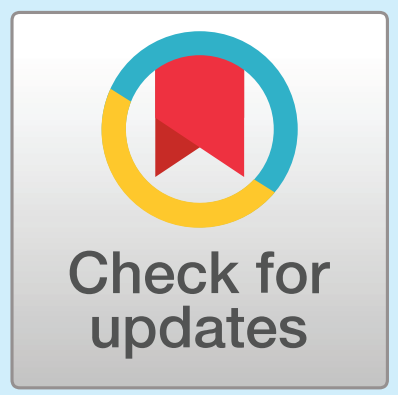

Citation: Degen, J., \& Tonhauser, J. (2021). Prior Beliefs Modulate Projection. Open Mind: Discoveries in Cognitive Science, 5, 59-70. https://doi .org/10.1162/opmi_a_00042

DOI:

https://doi.org/10.1162/opmi_a_00042

Supplemental Materials:

https://doi.org/10.1162/opmi_a_00042

Received: 19 February 2021

Accepted: 29 June 2021

Competing Interests: The authors declare that no conflict of interest.

Corresponding Author:

Judith Degen

jdegen@stanford.edu

Copyright: ๑ 2021

Massachusetts Institute of Technology Published under a Creative Commons Attribution 4.0 International (CC BY 4.0) license

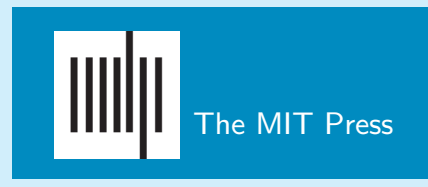

\begin{abstract}
Beliefs about the world affect language processing and interpretation in several empirical domains. In two experiments, we tested whether subjective prior beliefs about the probability of utterance content modulate projection, that is, listeners' inferences about speaker commitment to that content. We find that prior beliefs predict projection at both the group and the participant level: the higher the prior belief in a content, the more speakers are taken to be committed to it. This result motivates the integration of formal analyses of projection with cognitive theories of language understanding.
\end{abstract}

\section{INTRODUCTION}

Psycholinguistic work has documented several ways in which probabilistic beliefs about the world, often termed world knowledge, affect language processing (e.g., Chambers et al., 2002; Hagoort et al., 2004; Hald et al., 2007; Warren \& McConnell, 2007), including syntactic ambiguity resolution (e.g., Bicknell \& Rohde, 2014; Chambers et al., 2004), reference resolution (e.g., Hanna \& Tanenhaus, 2004; Winograd, 1972), genericity (e.g., Tessler \& Goodman, 2019), scalar implicature (e.g., Degen et al., 2015), underinformativity implicatures (Kravtchenko \& Demberg, 2015), and the production of redundant referring expressions (Degen et al., 2020; Mitchell et al., 2013; Rubio-Fernández, 2016; Sedivy, 2003; Westerbeek et al., 2015). In contrast, formal linguistic research on meaning in the tradition of Montague (1973), which is devoted to specifying how meanings of expressions are computed from the meanings of the parts of the expressions, the way the parts are combined, and the contexts in which the expressions are used, has often sidelined world knowledge as nonlinguistic, encyclopedic knowledge that must enter into the meaning computation, but whose effect has eluded systematic investigation and formalization (for relevant discussion see, e.g., Beaver, 2001; Dowty, 1986; Hobbs, 2019; Peeters, 2000). ${ }^{1}$ In this article, we provide empirical evidence from English that projection, a key topic in linguistic research on meaning, is systematically modulated by listeners ${ }^{\prime 2}$ subjective beliefs about the world. This provides further impetus

\footnotetext{
${ }^{1}$ Because knowledge implies justified true belief but subjective beliefs need not be accurate to affect language processing in systematic ways, we henceforth avoid the term world knowledge and instead refer to (subjective prior) beliefs about the world.

${ }^{2}$ We include readers, writers, and signers in the terms listener and speaker.
} 
for accounts of meaning computation to include a mechanism for integrating subjective prior beliefs. We provide a sketch of such an account at the end of this article.

To introduce projection, consider first that speakers can present themselves, through their utterances, as believing that a particular content is true, that is, as committed to that content. Listeners, in turn, regularly draw inferences about which content speakers present themselves as committed to. For instance, if a speaker utters Sam knows that it's raining, listeners typically infer that the speaker is committed to the following two contents: (i) the content of the complement (CC) of know, that it's raining; and (ii) the content of the matrix clause, that Sam knows (i). In formal research on meaning, the inference to (ii) is attributed to the speaker having uttered the sentence, and the inference to (i) is attributed to a particular aspect of the lexical meaning of know, specifically, that if an individual knows some content $p$, then $p$ is true (e.g., Chierchia \& McConnell-Ginet, 1990). The puzzle is that the inference to (i) may persist even when the speaker inquires about what Sam knows, as in Does Sam know that it's raining?, or when the speaker denies Sam's knowledge, as in Sam doesn't know that it's raining. Because Sam's knowledge is questioned or even denied in these variants, that is, the inference to (ii) does not persist, these inferences to (i) cannot be attributed to the aforementioned lexical meaning of know. This phenomenon of speaker commitment to utterance content that occurs in negated sentences or questions is termed projection. Decades of research in formal semantics have aimed to explain why content projects (e.g., Beaver \& Geurts, 2014; Langendoen \& Savin, 1971).

While content is standardly taken to either project or not (Beaver \& Geurts, 2014), recent experimental work suggests that projection is gradient: listeners' inferences about speaker commitment to utterance content vary in strength. This experimental work suggests that several factors modulate the strength of the inference, including the expression (e.g., know vs. discover vs. announce), the discourse status of the content, and the prosody of the utterance (for an overview see Tonhauser, Beaver, \& Degen, 2018). The hypothesis that listeners' prior beliefs modulate projection was initially put forth by Stevens et al. (2017) and Tonhauser et al. (2018), who observed by-item projection variability for different CCs of clause-embedding predicates like know and discover. They argued that one source of the observed variability may be that more a priori likely content (Kim flew to New York) projects more strongly than less a priori likely content (Kim flew to the moon) when realized as the CC of a clauseembedding predicate (as in Did John discover that Kim flew to New York/the moon?). This idea can straightforwardly be made sense of under recent Bayesian accounts that treat pragmatic utterance interpretation as a matter of combining uncertain prior beliefs about the world with uncertain beliefs about likely speaker production choices via Bayes' rule (Degen et al., 2015; Goodman \& Frank, 2016): a CC that is more likely a priori (before observing an utterance) is also more likely a posteriori (after observing an utterance).

There is conflicting evidence for the hypothesis that prior beliefs modulate projection. Support for the hypothesis comes from Mahler (2020), who investigated the projection of politically charged CCs of English clause-embedding predicates. For example, the politically charged content in (1) is that Obama improved/damaged the American economy. The prior probability of the content was manipulated by the speaker (Cindy in [1]) speaking at the club meeting of either the College Republicans or Democrats.

(1) Cindy, at the College Republicans/Democrats club meeting: Ben doesn't know that ...

a. ... Obama improved the American economy.

b. ... Obama damaged the American economy.

(Mahler, 2020, pp. 784-785) 
Higher prior probability content (e.g., a liberal content like [1a] uttered by a Democrat) was more projective than lower prior probability content (e.g., a liberal content uttered by a Republican).

In contrast, Lorson (2018) did not find empirical support for the hypothesis that listeners' prior beliefs modulate projection in a study of the projection of the prestate content of the English change of state verb stop. Prior probability was manipulated through gender stereotypes reported in Boyce, von der Malsburg, Poppels, and Levy (2018). For instance, because men are more likely than women to be plumbers, the prestate content of (2a), that James has worked as a plumber, was hypothesized to be more projective than the prestate content of (2b), that Linda has worked as a plumber.

(2) a. Did James stop working as a plumber?

b. Did Linda stop working as a plumber?

(Lorson, 2018, p. 38)

Several differences between Mahler (2020) and Lorson (2018) could be implicated in the differential support for the hypothesis: (a) the projective content investigated (CCs vs. prestate content of stop); (b) stimulus type (negated sentences vs. questions); (c) the manipulation of prior beliefs (political party affiliation vs. gender stereotypes); and (d) how explicitly the prior-manipulating information was provided to participants (statement of political party affiliation vs. use of a male or female name to indicate gender). The two experiments reported on in this article provide additional support for the hypothesis that prior beliefs modulate projection. The experiments included 20 clause-embedding predicates (rather than just 7 , as in Mahler, 2020) and the prior belief manipulation involved 20 properties of individuals, rather than just political party affiliation (as in Mahler, 2020), or gender (as in Lorson, 2018). Furthermore, we tested the hypothesis both at the level of the individual and of the group: Experiment 1 investigated the effect of prior beliefs on projection by measuring prior probability and projection in a within-participant design. In Experiments 2a and 2b, prior probability and projection were measured in separate groups, as in Mahler (2020) and Lorson (2018).

\section{EXPERIMENT 1}

This experiment tested whether higher prior probability content is more likely to project than lower probability content. Prior probability and projection ratings were collected for the contents of 20 clauses that realized the complements of 20 clause-embedding predicates. ${ }^{3}$

\section{Methods}

Participants Three hundred participants with U.S. IP addresses and at least $99 \%$ of previous human intelligence tasks (HITs) approved were recruited on Amazon's Mechanical Turk platform (ages: 18-82, median: 35.5; 119 female, 179 male, 1 other, 1 undeclared). They were paid $\$ 1.80$ and took 9.1 minutes on average to complete the experiment.

\footnotetext{
3 The experiments, data and $\mathrm{R}$ code for generating the figures and analyses of the experiments reported on in this article are available at https://github.com/judith-tonhauser/projective-probability. Experiment 1 was preregistered: https://osf.io/vd9ru/. All experiments were conducted with approval from the institutional review board of The Ohio State University and informed consent was obtained.
} 

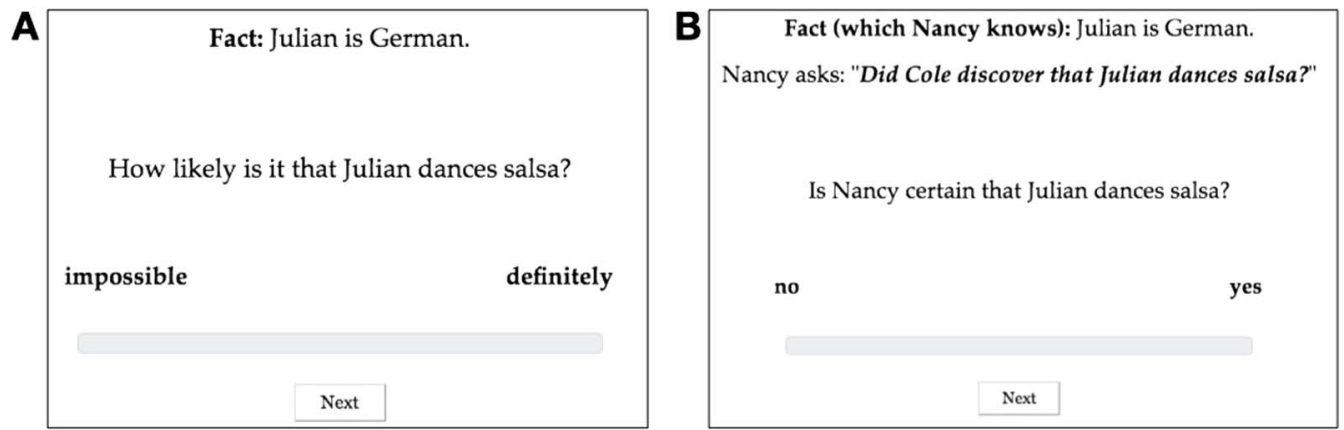

acknowledge, admit, announce, be annoyed, be right, confess, confirm, demonstrate, discover, establish, hear, inform, know, pretend, prove, reveal, say, see, suggest, think.
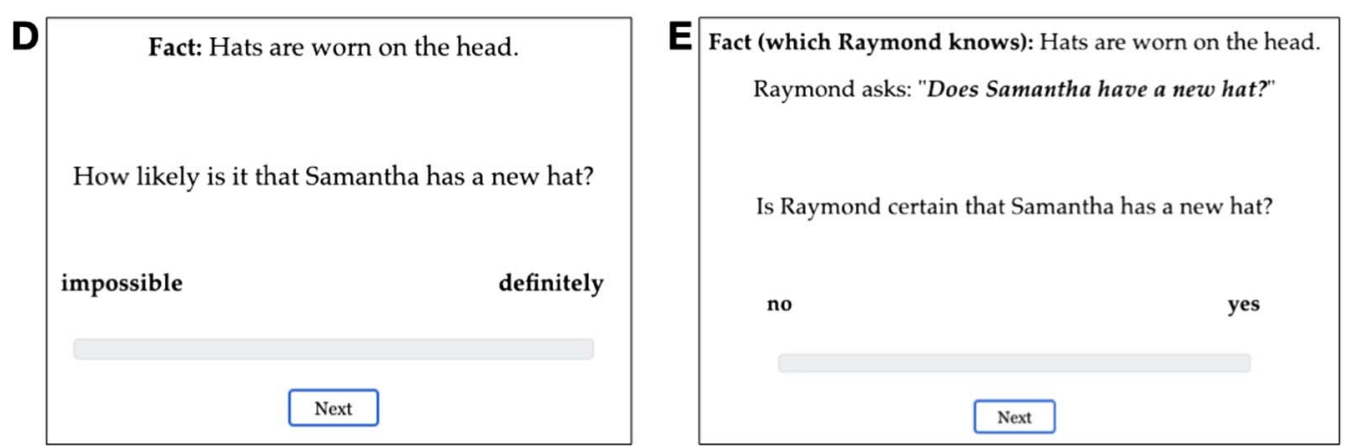

Figure 1. Example trials and 20 clause-embedding predicates. A. Example target trial in prior block. B. Example target trial in projection block. C. The 20 clause-embedding predicates. D. Example filler trial in prior block. E. Example control trial in projection block.

Materials and Procedure The prior probability and projection of the contents of 20 clauses were measured in separate blocks. Each clause (e.g., Julian dances salsa) was paired with two facts between participants: The content of the clause was expected to have a higher prior probability in the presence of one fact (e.g., Julian is Cuban) than of the other (e.g., Julian is German). See the Supplemental Materials for the full set of clauses and facts.

In the prior block, the 20 clauses were realized as the complements of How likely is it that ... ? questions. As shown in Figure 1A, each target stimulus consisted of one of the two facts for that clause and the How likely is it that ... ? question. Participants read the fact and assessed the likelihood of the content, given the fact. They gave their responses on a slider marked "impossible" at one end (coded as 0) and "definitely" at the other (coded as 1).

In the projection block, target stimuli consisted of a fact and a polar question that was uttered by a named speaker, as shown in Figure 1B. The polar questions were formed by realizing the 20 clauses as the complements of the 20 clause-embedding predicates in Figure 1C. Participants were told to imagine that they are at a party and that, on walking into the kitchen, they overhear somebody ask somebody else a question. Projection was measured using the "certain that" diagnostic (Djärv \& Bacovcin, 2017; Lorson, 2018; Mahler, 2020; Tonhauser et al., 2018): participants were asked to rate whether the speaker was certain of the CC, taking into consideration the fact. They gave their responses on a slider marked "no" at one end (coded as 0 ) and "yes" at the other (coded as 1). Greater speaker commitment to the CC should result in higher slider ratings.

The projection block also included six control trials, which functioned as attention checks. The content of these stimuli was expected not to project: For example, in Figure 1E, the 


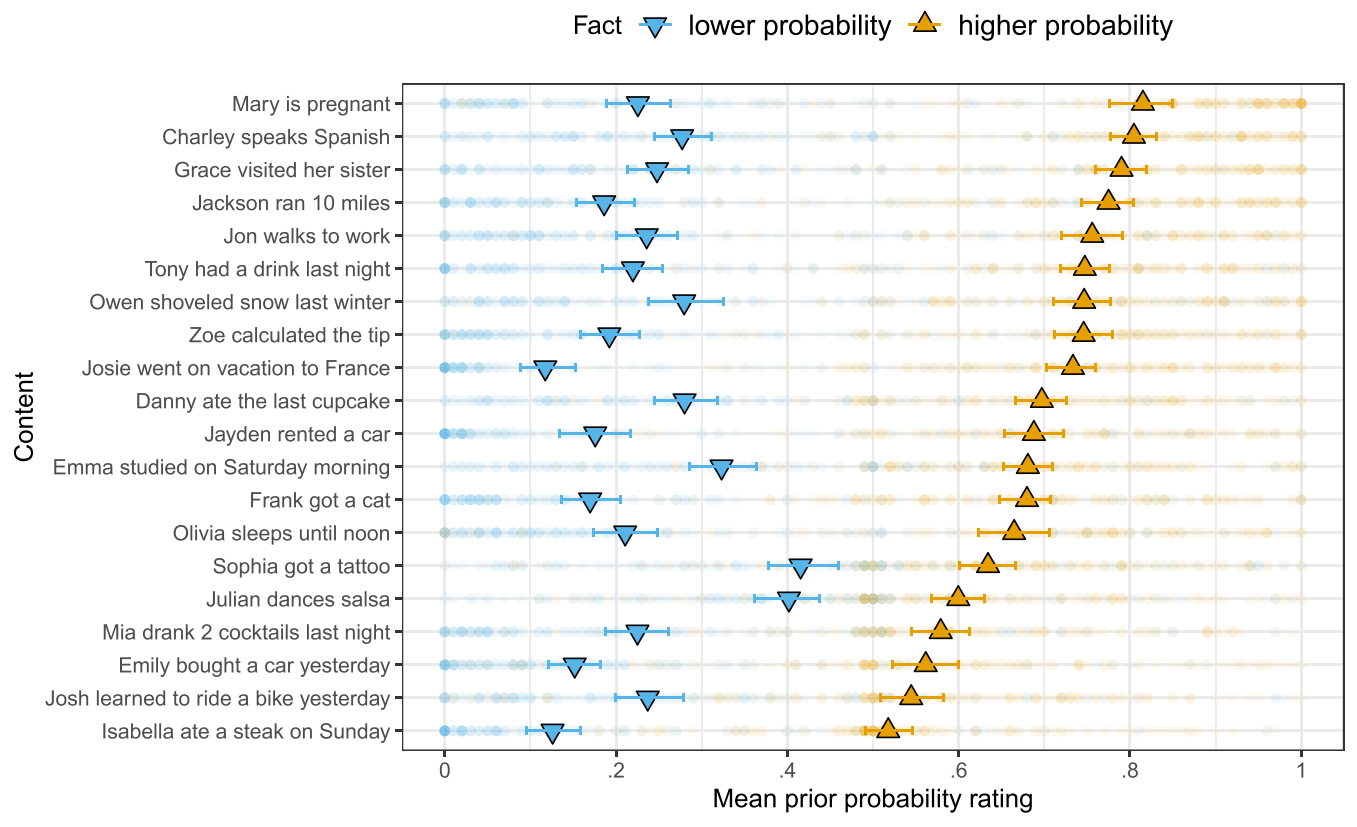

Figure 2. Mean prior probability by content and fact in Experiment 1. Error bars indicate 95\% bootstrapped confidence intervals. Transparent dots indicate individual participant ratings.

speaker is not committed to the main clause content, that Samantha has a new hat. The same six main clauses were also used to form 6 filler trials in the prior block; a sample stimulus is given in Figure 1D. These filler stimuli were not used to assess participants' attention. For the full set of stimuli see the Supplemental Materials.

Each participant's stimulus set was semi-randomly generated by first randomly pairing up the 20 predicates and clauses. Half of the stimuli were then randomly assigned the respective clause's higher-probability fact, and half its lower-probability fact. Participants completed a total of 52 trials: 20 target trials in each block, six control trials in the projection block, and six filler trials in the prior block. Each participant completed the same six filler and control trials. Block order and within-block trial order were randomized.

After completing the experiment, participants filled out a short optional demographic survey. To encourage truthful responses, participants were told that they would be paid no matter what answers they gave in the survey.

Data Exclusion Data were excluded based on self-declared non-native speaker status and other criteria given in the Supplemental Materials, leaving 5,720 data points from 286 participants to be analyzed (ages 18-82; median: 35.5; 116 female, 186 male, 1 other, 1 undeclared).

\section{Results and Discussion}

Prior Beliefs Figure 2 shows the mean prior probabilities of the 20 contents by fact. We conducted a mixed-effects linear regression predicting slider rating from dummy-coded fact type (reference level: "lower probability") and random by-item and by-participant intercepts and slopes for fact type. ${ }^{4}$ Each content's mean prior probability was rated as higher when it was presented with its higher probability fact than when it was presented with its lower probability

\footnotetext{
${ }^{4}$ All analyses were conducted in R (R Core Team, 2016) using the lme 4 package (Bates et al., 2015).
} 


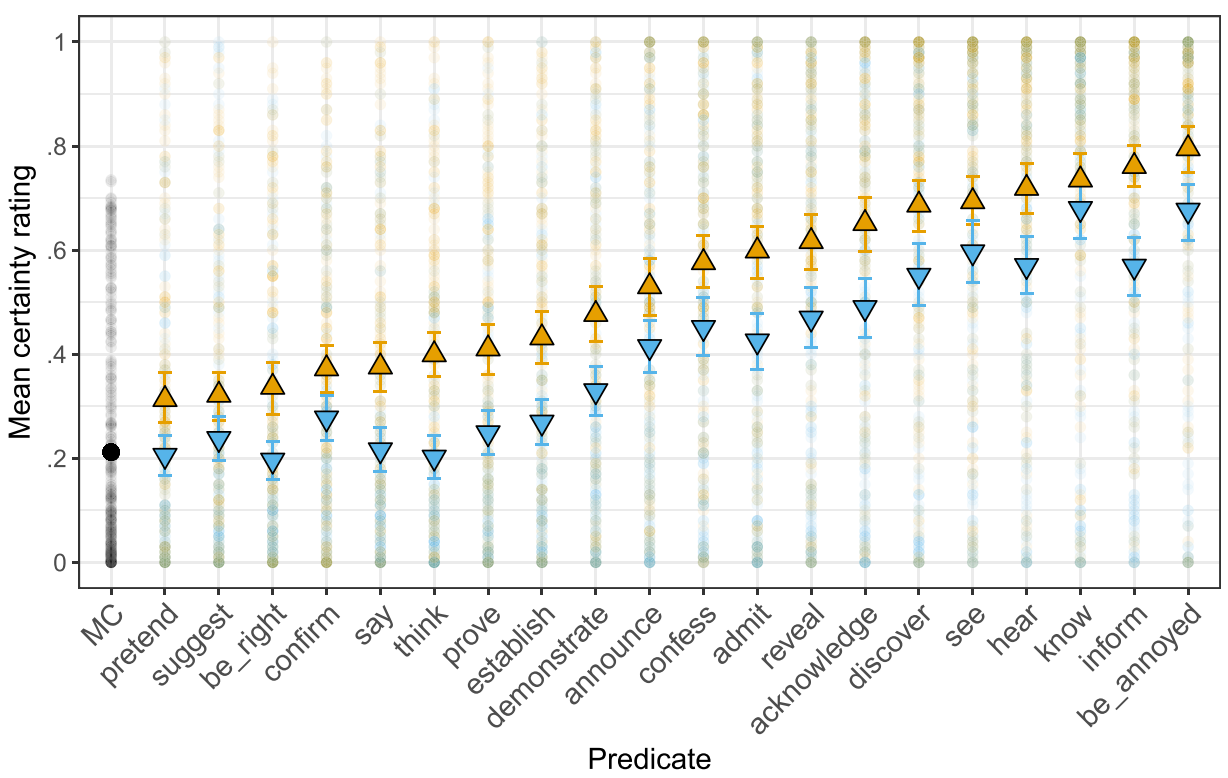

Figure 3. Mean certainty ratings by predicate and prior probability of the content of the complement in Experiment 1. Error bars indicate 95\% bootstrapped confidence intervals. Light dots indicate participants' ratings.

fact $(\beta=0.45, S E=0.01, t=31.12, p<.0001)$. This suggests that the manipulation of the prior probability of the 20 contents was successful.

Do Prior Beliefs Modulate Projection? Figure 3 shows the mean certainty ratings for the CCs by predicate and by fact, as well as the mean certainty rating for the main clause controls (abbreviated MC). Each predicate/clause combination was rated 5-25 times (mean 14.3). We conducted a mixed effects linear regression predicting certainty ratings from dummy-coded fact type (reference level: "lower probability") and random by-item and by-participant intercepts and slopes for fact type. The mean certainty ratings were higher for contents presented with higher probability facts than for contents presented with lower probability facts $(\beta=0.14, S E=$ $0.01, t=12.24, p<.0001)$. The same was true when using the group-level by-item mean prior belief as a predictor $(\beta=0.31, S E=0.02, t=12.58, p<.0001)$. This suggests that participants ${ }^{\prime}$ prior beliefs about content probability systematically modulated the extent to which they take the speaker to be committed to that content.

We also replicated the by-predicate variability in the projection of the CC observed by Tonhauser and Degen (2020): for instance, the CC of be annoyed was more projective than that of discover, which in turn was more projective than that of announce. The Spearman rank correlation between the mean certainty ratings in Experiment 1 (collapsing over facts) and Experiment 1a of Tonhauser and Degen (2020) is .991; see the Supplemental Materials for a visualization. Experiment 1 thereby also provides further evidence for the systematic influence of the predicate on projection. Crucially, the effect of the prior was observable independently of the predicate.

Closer inspection of Figure 2 reveals by-participant variability in prior probability ratings, suggesting that individual participants' prior beliefs may not align with the prior probability classification assumed in Figure 3. For example, given a particular content (Julian dances 


\section{Fact - lower probability $\bullet$ higher probability}
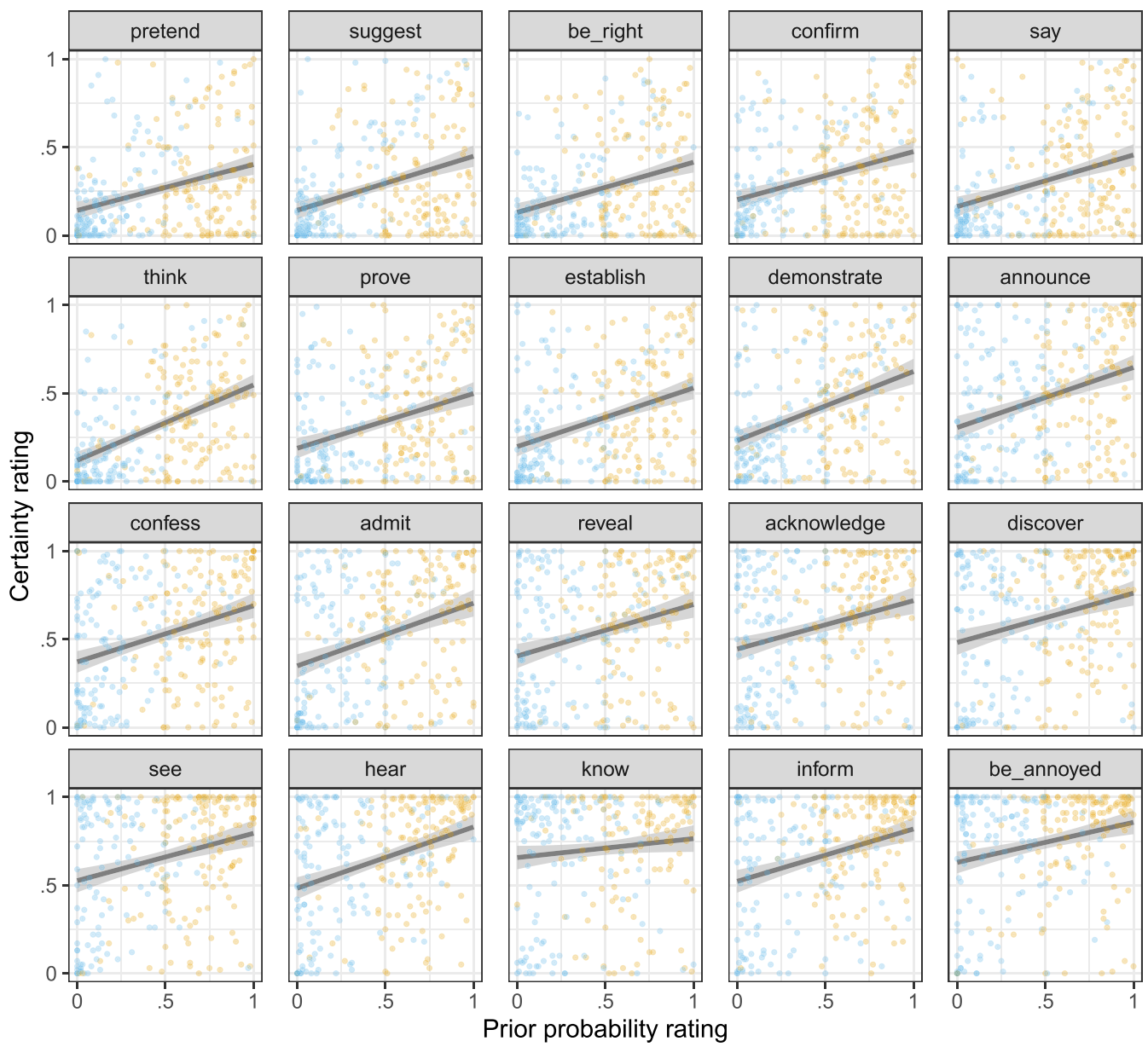

Figure 4. Certainty ratings against individual prior probability ratings for each predicate in Experiment 1 . Linear smoothers with $95 \%$ confidence intervals are overlaid.

salsa), it is possible that one participant's prior probability rating was lower than that of another participant, even though the first participant was presented with the higher probability fact (Julian is Cuban) and the second one with the lower probability fact (Julian is German). Figure 4 shows participants' certainty ratings by their individual prior probability ratings. To investigate whether prior beliefs modulate projection at the by-participant level, we conducted the same mixed-effects analysis reported above, but used participants' individual, continuous prior probability ratings as the fixed effect prior predictor. Again, higher prior-probability CCs were more likely to project $(\beta=0.28, S E=0.02, t=13.85, p<.0001$ ). This suggests that prior beliefs modulate projection even at the by-participant level. A Bayesian information criterion (BIC) model comparison revealed that the individual-level model better captured the variance in the data (categorical model BIC: 2654; group-level model BIC: 2586; individual-level model BIC: 2291), ${ }^{5}$ suggesting that individual listeners' prior beliefs systematically modulate the

\footnotetext{
${ }^{5}$ The BIC model comparisons were not preregistered, so we also ran Akaike information criterion (AIC) model comparisons as a robustness check. The results were qualitatively identical (categorical model AIC: 2607; group-level model AIC: 2539; individual-level model AIC: 2244).
} 
extent to which they take the speaker to be committed to a content: the more they believe it, the more they take the speaker to believe it.

The results of Experiment 1 provide empirical support for the hypothesis that higher prior probability content is more likely to project. It is possible, however, that the within-participant design resulted in participants' responses on either block influencing their responses on the other block. To guard against this possibility, we replicated Experiment 1 by collecting prior probability and projection ratings from different groups.

\section{EXPERIMENT 2}

Experiments $2 \mathrm{a}$ and $2 \mathrm{~b}$ measured the prior probability and the projection of the 20 contents of Experiment 1, respectively.

\section{Methods}

Participants Participants with U.S. IP addresses and at least $99 \%$ of previous HITs approved were recruited on Amazon's Mechanical Turk platform. The 95 participants in Experiment 2a (ages: $21-75$, median: 33; 45 female, 50 male) were paid 55 cents and took 3.9 minutes on average to complete the experiment. The 300 participants in Experiment $2 b$ (ages: 21-72, median: 36; 145 female, 154 male, 1 undeclared) were paid 85 cents and took 7.1 minutes on average.

Materials and Procedures The target stimuli of Experiment 2a were identical to those of the prior block of Experiment 1. Each participant saw two control stimuli as attention checks (see the Supplemental Materials). The materials of Experiment $2 \mathrm{~b}$ were identical to those of the projection block of Experiment 1. Trial order in both experiments was random. The procedures of Experiments $2 \mathrm{a}$ and $2 \mathrm{~b}$ were identical to those of the prior and projection blocks of Experiment 1, respectively.

Data Exclusion We excluded data based on the criteria given in the Supplemental Materials, leaving data from 75 participants to be analyzed in Experiment 2a (1,500 data points; ages 2175; median: 35; 34 female, 41 male) and from 266 participants in Experiment 2b (5,320 data points; ages 21-72; median: 36; 129 female, 136 male, 1 undeclared).

\section{Results and Discussion}

Prior Beliefs Experiment 2a successfully replicated the prior probability manipulation of Experiment 1: contents were rated as more likely when presented with a higher probability fact $(\beta=0.54, S E=0.04, t=15.07, p<.0001$ ). Figure 5 shows contents' mean prior probability ratings in Experiment 2a against those of Experiment 1. The Spearman rank correlation was very high, at $r=.977$. For a visualization of the by-content prior ratings see the Supplemental Materials.

Do Prior Beliefs Modulate Projection? Each predicate/clause combination was rated 4-27 times (mean 13.3). Mean certainty ratings were higher for contents presented with higher prior probability facts than for contents presented with lower prior probability facts (see Figure 6). This was true when the prior predictor was entered as a categorical predictor (reference level: "lower probability"; $\beta=0.18, S E=0.01, t=12.81, p<.0001$ ) and when it was entered as a continuous predictor representing group-level prior means $(\beta=0.34, S E=0.03, t=13.27, p<.0001)$. Thus, Experiment $2 \mathrm{~b}$ replicates the critical result of Experiment 1 that prior content probability 
Fact $\nabla$ lower probability $\triangle$ higher probability

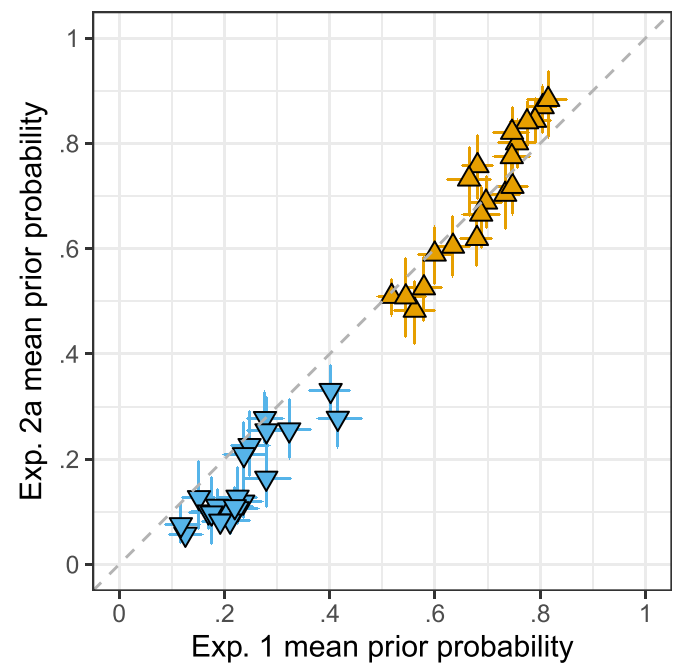

Figure 5. Mean prior probability ratings in Experiment 2a against those of Experiment 1. Error bars indicate $95 \%$ bootstrapped confidence intervals.

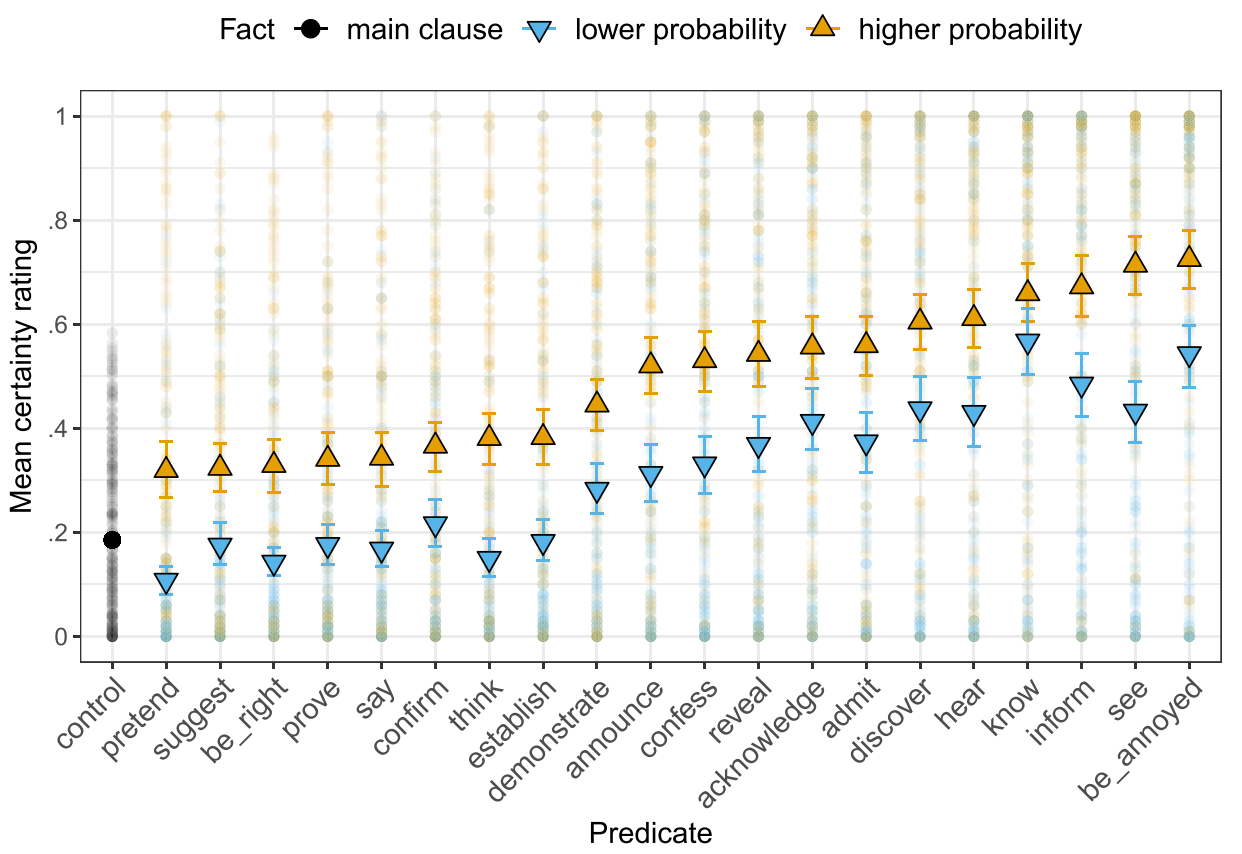

Figure 6. Mean certainty ratings by predicate and prior probability of the content of the complement in Experiment 2b. Error bars indicate 95\% bootstrapped confidence intervals. Light dots indicate participants' ratings. 
modulates its projection. ${ }^{6}$ The replication suggests that the result of Experiment 1 is not an artifact of the within-participant design of Experiment 1.

\section{GENERAL DISCUSSION AND CONCLUDING REMARKS}

We tested whether listeners' prior beliefs modulate projection. While previous research on this question has yielded conflicting results (Lorson, 2018; Mahler, 2020), we showed in two experiments that content is more likely to project the more a priori likely it is, thus confirming the results of Mahler (2020) and expanding on them in several ways. First, while Mahler (2020) manipulated only the political party affiliation of the speaker, the manipulation in Experiments 1 and 2 relied on 20 distinct properties of individuals (e.g., whether Julian is more likely to dance salsa if he is German or Cuban). Thus, the results of Experiments 1 and 2 suggest a general effect of prior beliefs on projection. Second, our experiments show that prior beliefs modulate projection for a wider cross-section of clause-embedding predicates, including cognitive (e.g., know), emotive (e.g., be annoyed), communication (e.g., announce), and inferential (e.g., prove) predicates. Thus, projection joins the many linguistic phenomena that are systematically modulated by prior beliefs (Bicknell \& Rohde, 2014; Chambers et al., 2004; Degen et al., 2015; Hagoort et al., 2004; Hald et al., 2007; Kravtchenko \& Demberg, 2015; Sedivy, 2003; Tessler \& Goodman, 2019; Warren \& McConnell, 2007; Westerbeek et al., 2015). Finally, the within-participant design of Experiment 1 shows that individuals' gradient prior beliefs better predict projection than gradient group-level beliefs, which in tum better predict projection than binary, categorical group-level beliefs (as investigated by Mahler, 2020). This suggests that at least some by-participant variability observed in previous projection experiments (see, e.g., Tonhauser et al., 2018; Tonhauser \& Degen, 2020) may be due to participants assigning different prior probabilities to investigated content.

Does our work help explain the discrepancy in findings between the work of Mahler (2020) and Lorson (2018)? In the introduction, we raised four possibilities for the observed differences: (a) the projective content investigated (CCs vs. prestate content of stop); (b) stimulus type (negated sentences vs. questions); (c) the manipulation of prior beliefs (political party affiliation vs. gender stereotypes); and (d) how explicitly the prior-manipulating information was provided to participants (statement of political party affiliation vs. use of a male or female name to indicate gender). Our work used (a) CCs; (b) questions; (c) introduced novel properties of individuals; and (d) introduced prior-manipulating information via explicit statement of facts. Thus, our work rules out option (b) - that prior beliefs don't affect projection out of questions-but not the other possibilities, which should be investigated in future work.

Our results have two broader implications. First, they suggest that the purview of projection analyses is wider than assumed by current analyses, which typically limit their attention to a narrow subset of clause-embedding predicates, like factive ones (e.g., Abrusán, 2011, 2016; Heim, 1983; Romoli, 2015; Simons et al., 2017; van der Sandt, 1992). Rather than being categorical and limited to factive predicates, projection inferences are systematically drawn to varying degrees as a function of at least predicate identity and prior beliefs about content. Second, the results motivate the development of projection analyses that consider listeners' variable subjective beliefs about the world. Given the gradient nature of the measured (prior and posterior) beliefs and the uncertainty inherent in the different factors that have been shown to modulate projection (e.g., at-issueness, prosody), probability theory suggests itself as a

\footnotetext{
${ }^{6} 28$ participants took Experiment 2b after taking Experiment 2a two weeks before. Analyses that excluded these participants' data did not change the results. Experiment $2 \mathrm{~b}$ also replicated the result of Tonhauser and Degen (2020) that there is by-predicate variability in the projection of the CC; see the Supplemental Materials.
} 
representational framework within which to model projection. To date, only few probabilistic models of projection have been developed (Qing, Goodman, \& Lassiter, 2016; Stevens et al., 2017). In these models, projection is the result of listeners' reasoning about the common ground that the speaker is assuming and the likely question that was being addressed, respectively. While neither investigated the effect of prior beliefs explicitly, both models are couched within the Rational Speech Act (RSA) framework (Franke \& Jäger, 2016; Goodman \& Frank, 2016), which standardly assumes that utterance interpretation is modulated by listeners' prior beliefs. The RSA framework is thus equipped to capture the effects reported here. We see the implementation of projection analyses within RSA as a promising avenue for formalizing the intricate interplay of semantic and pragmatic factors in the projection of contents of complements of clause-embedding predicates, including the conventional contribution of predicates, content at-issueness, and subjective prior beliefs about content.

\section{ACKNOWLEDGMENTS}

For helpful comments on the research presented here, we thank the audiences at the 2018 Annual Meeting of XPRAG.de and at the University of Tübingen.

\section{FUNDING INFORMATION}

JT, National Science Foundation (https://dx.doi.org/10.13039/100000001), Award ID: BCS1452674.

\section{AUTHOR CONTRIBUTIONS}

JD: Conceptualization: Equal; Formal analysis: Lead; Methodology: Equal; Visualization: Lead; Writing - Original Draft: Equal; Writing - Review \& Editing: Equal. JT: Conceptualization: Equal; Formal analysis: Supporting; Methodology: Equal; Visualization: Supporting; Writing Original Draft: Equal; Writing - Review \& Editing: Equal.

\section{REFERENCES}

Abrusán, M. (2011). Predicting the presuppositions of soft triggers. Linguistics \& Philosophy, 34(6), 491-535. https://doi.org/10.1007 /s10988-012-9108-y

Abrusán, M. (2016). Presupposition cancellation: Explaining the "soft-hard" trigger distinction. Natural Language Semantics, 24(2), 165-202. https://doi.org/10.1007/s11050-016-9122-7

Bates, D., Mächler, M., Bolker, B., \& Walker, S. (2015). Fitting linear mixed-effects models using Ime4. Journal of Statistical Software, 67(1), 1-48. https://doi.org/10.18637/jss.v067.i01

Beaver, D. (2001). Presupposition and assertion in dynamic semantics. CSLI Publications.

Beaver, D., \& Geurts, B. (2014). Presupposition. In E. Zalta (Ed.), The Stanford Encyclopedia of Philosophy. Stanford University.

Bicknell, K., \& Rohde, H. (2014). Dynamic integration of pragmatic expectations and real-world event knowledge in syntactic ambiguity resolution. In Proceedings of the 31st Annual Conference of the Cognitive Science Society (pp. 1216-1221). Cognitive Science Society.

Boyce, V., von der Malsburg, T., Poppels, T., \& Levy, R. (2018). Implicit gender in the production and comprehension of pronominal references. In F. Ferreira, J. Henderson, T. Swaab, \&
M. Traxler (Eds.), Proceedings of the 31st Annual CUNY Conference on Human Sentence Processing. UC Davis.

Chambers, C., Tanenhaus, M., Eberhard, K., Filip, H., \& Carlson, G. (2002). Circumscribing referential domains during real-time language comprehension. Journal of Memory and Language, 47(1), 30-49. https://doi.org/10.1006/jmla.2001.2832

Chambers, C., Tanenhaus, M., \& Magnuson, J. (2004). Actions and affordances in syntactic ambiguity resolution. Journal of Experimental Psychology, 30(3), 687-696. https://doi.org/10 1037/0278-7393.30.3.687, PubMed: 15099136

Chierchia, G., \& McConnell-Ginet, S. (1990). Meaning and grammar. MIT Press.

Degen, J., Hawkins, R. X. D., Graf, C., Kreiss, E., \& Goodman, N. D. (2020). When redundancy is useful: A Bayesian approach to "overinformative" referring expressions. Psychological Review, 127(4), 591-621. https://doi.org/10.1037/rev0000186, PubMed: 32237876

Degen, J., Tessler, M. H., \& Goodman, N. D. (2015). Wonky worlds: Listeners revise world knowledge when utterances are odd. In D. C. Noelle, R. Dale, A. S. Warlaumont, J. Yoshimi, T. Matlock, C. D. Jennings, \& P. P. Maglio (Eds.), Proceedings of 
the 37th Annual Conference of the Cognitive Science Society (pp. 548-553). Cognitive Science Society.

Djärv, K., \& Bacovcin, H. (2017). Prosodic effects on factive presupposition projection. Semantics and Linguistic Theory, 27, 116-133. https://doi.org/10.3765/salt.v27i0.4134

Dowty, D. (1986). The effects of aspectual class on the temporal structure of discourse: Semantics or pragmatics? Linguistics \& Philosophy, 9(1), 37-61.

Franke, M., \& Jäger, G. (2016). Probabilistic pragmatics, or why Bayes' rule is probably important for pragmatics. Zeitschrift für Sprachwissenschaft, 35(1), 3-44. https://doi.org/10.1515/zfs -2016-0002

Goodman, N. D., \& Frank, M. C. (2016). Pragmatic language interpretation as probabilistic inference. Trends in Cognitive Sciences, 20(11), 818-829. https://doi.org/10.1016/j.tics.2016.08.005, PubMed: 27692852

Hagoort, P., Hald, L., Mastiaansen, M., \& Petersson, K. (2004). Integration of word meaning and world knowledge in language comprehension. Science, 304(5669), 438-441. https://doi.org/10 .1126/science.1095455, PubMed: 15031438

Hald, L. A., Steenbeek-Planting, E., \& Hagoort, P. (2007). The interaction of discourse context and world knowledge in online sentence comprehension. Evidence from the N400. Brain Research, 1146(2007), 210-218. https://doi.org/10.1016/j.brainres.2007 .02.054, PubMed: 17433893

Hanna, J., \& Tanenhaus, M. (2004). Pragmatic effects on reference resolution in a collaborative task: Evidence from eye movements. Cognitive Science, 28, 105-115. https://doi.org/10.1207 /s15516709cog2801_5

Heim, I. (1983). On the projection problem for presuppositions. West Coast Conference on Formal Linguistics, 2, 114-125.

Hobbs, J. (2019). Word meaning and world knowledge. In C. Maienborn, K. von Heusinger, \& P. Portner (Eds.), Semantics (pp. 154-180). de Gruyter. https://doi.org/10.1515 /9783110589245-006

Kravtchenko, E., \& Demberg, V. (2015). Semantically underinformative utterances trigger pragmatic inferences. In D. C. Noelle, R. Dale, A. S. Warlaumont, J. Yoshimi, T. Matlock, C. D. Jennings, \& P. P. Maglio (Eds.), Proceedings of the 37th Annual Conference of the Cognitive Science Society (pp. 1207-1212). Cognitive Science Society.

Langendoen, T., \& Savin, H. (1971). The projection problem for presuppositions. In C. Fillmore \& T. Langendoen (Eds.), Studies in linguistic semantics (pp. 54-60). Holt, Rinehart and Winston.

Lorson, A. (2018). The influence of world knowledge on projectivity (Master's thesis, University of Potsdam).

Mahler, T. (2020). The social component of projection behavior of clausal complements. Linguistic Society of America, 5(1), 777-791. https://doi.org/10.3765/plsa.v5i1.4703

Mitchell, M., Reiter, E., \& Van Deemter, K. (2013). Typicality and object reference. In Proceedings of the 35th Annual Meeting of the Cognitive Science Society (pp. 3062-3067). Cognitive Science Society.

Montague, R. (1973). The proper treatment of quantification in ordinary English. In J. Hintikka, J. Moravcsik, \& P. Suppes (Eds.), Approaches to natural language: Proceedings of the 1970
Stanford workshop on grammar and semantics. Reidel. https:// doi.org/10.1007/978-94-010-2506-5 10

Peeters, B. (2000). Setting the scene: Some recent milestones in the lexicon-encyclopedia debate. In B. Peeters (Ed.), The lexiconencyclopedia interface (pp. 1-52). Elsevier.

Qing, C., Goodman, N. D., \& Lassiter, D. (2016). A rational speech-act model of projective content. In A. Papafragou, D. Grodner, D. Mirman, \& J. C. Trueswell (Eds.), Proceedings of the 38th Annual Conference of the Cognitive Science Society (pp. 1110-1115). Cognitive Science Society.

R Core Team. (2016). R: A language and environment for statistical computing [Computer software manual]. https://www.R-project.org/

Romoli, J. (2015). The presuppositions of soft triggers are obligatory scalar implicatures. Journal of Semantics, 32(2), 173-291. https:// doi.org/10.1093/jos/fft017

Rubio-Fernández, P. (2016). How redundant are redundant color adjectives? An efficiency-based analysis of color overspecification. Frontiers in Psychology, 7. https://doi.org/10.3389 /fpsyg.2016.00153, PubMed: 26924999

Sedivy, J. C. (2003). Pragmatic versus form-based accounts of referential contrast: Evidence for effects of informativity expectations. Journal of Psycholinguistic Research, 32(1), 3-23. https://doi.org 10.1023/A:1021928914454, PubMed: 12647560

Simons, M., Beaver, D., Roberts, C., \& Tonhauser, J. (2017). The best question: Explaining the projection behavior of factive verbs. Discourse Processes, 54(3), 187-206. https://doi.org/10 .1080/0163853X.2016.1150660

Stevens, J., de Marneffe, M.-C., Speer, S., \& Tonhauser, J. (2017). Rational use of prosody predicts projectivity in manner adverb utterances. Annual Meeting of the Cognitive Science Society, 39, 1144-1149.

Tessler, M. H., \& Goodman, N. (2019). The language of generalization. Psychological Review, 126(3), 395-436. https://doi.org /10.1037/rev0000142, PubMed: 30762385

Tonhauser, J., Beaver, D., \& Degen, J. (2018). How projective is projective content? Gradience in projectivity and at-issueness. Journal of Semantics, 35(3), 495-542. https://doi.org/10.1093/jos/ffy007

Tonhauser, J., \& Degen, J. (2020). Are there factive predicates? An empirical investigation (Manuscript submitted for publication). Stuttgart University and Stanford University. https://ling.auf.net /lingbuzz/005360

van der Sandt, R. (1992). Presupposition projection as anaphora resolution. Journal of Semantics, 9(4), 333-377. https://doi.org /10.1093/jos/9.4.333

Warren, T., \& McConnell, K. (2007). Investigating effects of selectional restriction violations and plausibility violation severity on eye-movements in reading. Psychonomic Bulletin \& Review, 14(4), 770-775. https://doi.org/10.3758/BF03196835, PubMed: 17972747

Westerbeek, H., Koolen, R., \& Maes, A. (2015). Stored object knowledge and the production of referring expressions: The case of color typicality. Frontiers in Psychology, 6. https://doi.org/10 3389/fpsyg.2015.00935, PubMed: 26217268

Winograd, T. (1972). Understanding natural language. Cognitive Psychology, 3(1), 1-191. https://doi.org/10.1016/0010-0285(72) 90002-3 